12) Soo, S. L. and N. J. Princeton: Trans $A S M E, 80,287$ (1958).

13) Szeri, A. Z., S. J. Schneider and F. Labbe: J. Fluid Mech., 134, 103 (1983).

14) Tiller, F. M. and K. S. Chemg: Filtr. \& Separ., 14, 13 (1977).

15) Toda, T.: Filtration Symposium Nagoya 86, Japan, 214
(1986).

16) Wilson, L. O. and N. L. Schryer: J. Fluid Mech., 85, 479 (1978)

17) Wroński, S. and A. Mróz: Filtr. \& Separ., 21, 397 (1984)

\title{
DESIGN OF HEAT EXCHANGER NETWORK WITH UNCERTAINTY IN OVERALL HEAT TRANSFER COEFFICIENTS
}

\author{
HIROKAZU NISHITANI, YOSHINORI KUTSUWA, KOUJI SHIMIZU, \\ AND EIICHI KUNUGITA \\ Department of Chemical Engineering, Osaka University, Toyonaka 560
}

Key Words: Process System, Procèss Design, Uncertainty, Tolerance, Heat Exchanger Network, Heat Transfer Coefficient

\begin{abstract}
Any heat exchanger network has an inherent region of tolerance for variations in the overall heat transfer coefficients. A simple rule on shift of the inherent region of tolerance by redesign of each heat transfer area has been extracted from the characteristics of the system model. This property allows development of a graphical design method for a flexible heat exchanger network with a specified region of required tolerance. The design method is an iterative one composed of a feasibility test of the proposed design for the specified region of required tolerance and a noninferiority test of the design for optimality. As an illustration, a noninferior solution with feasibility in the specified orthotope was effectively obtained by the proposed design method for acyclic or cyclic heat exchanger networks.
\end{abstract}

\section{Introduction}

Rational design in the face of uncertainties of the plant parameters is one of the most important engineering problems. To cope with these uncertainties, design margins have been determined by designers. They know from experience that a uniform design margin for every process unit does not always make sense. But they have not had a systematic approach to setting the right margin for a given unit. Recently the situation has been changing. Variations in plant parameters can be identified quantitatively by using computer data analysis. This enables representation of the uncertainties by the variations of uncertain parameters in the models. As a result, the specification on system flexibility can be formulated at the stage of process design.

Design problems to guarantee the feasibility of operation for any variation in the specified region of uncertain parameters have been studied by many researchers. ${ }^{1-6,8)}$ In principle the design problem can be solved when the region of required tolerance is

\footnotetext{
Received September 21, 1987. Correspondence concorning this article should be addressed to H. Nishitani. Y. Kutsuwa is now with Department of Chemical Engincering, Kyushu University.
}

given by an orthotope and the inherent region of tolerance of the system is composed of a finite set of convex or one-dimensional convex regions. ${ }^{6)}$ However, solving the design problem requires enormous computation efforts. Therefore, iterative design procedures have been applied to solve the problem.

From the geometrical viewpoint the problem is to obtain a design such that the inherent region of tolerance of the system contains the region of required tolerance in the uncertain parameter space. Generally, interaction between design and location of the inherent region of tolerance of the system is very complicated. This makes the systematic determination of design margins difficult.

In this paper it is shown that in the heat exchanger network a simple relationship exists between redesign of sizing and shift of the inherent region of tolerance of the system for the very common uncertainty of the overall heat transfer coefficients. This property is applied to the design of a network with a specified region of required tolerance.

\section{Heat Exchanger Network}

A heat exchanger network is composed of heat exchange units and auxiliary heaters and/or coolers as 


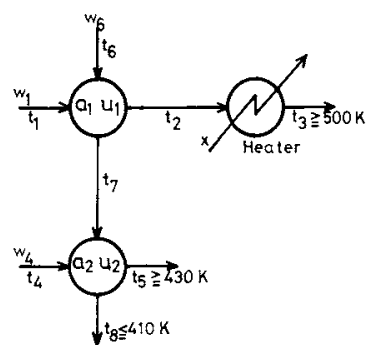

Fig. 1. An acyclic network with 2 countercurrent heat exchangers and a manipulated variable $x$ : example 1

shown in Fig. 1. The system equations for a unit can be derived from both the heat balance and the heat transfer rate equations. They can be represented by the linear equations between inlet and outlet temperatures of both the cold and hot streams. ${ }^{9}{ }^{\text {f }}$

$$
\left.\begin{array}{l}
t_{c o}=(1-e) t_{c i}+(e) t_{h i} \\
t_{h o}=(e r) t_{c i}+(1-e r) t_{h i}
\end{array}\right\}
$$

where $r$ is the ratio of heat capacity flow-rates of the two streams, i.e. $r=w_{c} / w_{h}$, and $e$ is the heat exchanger effectiveness. Once the type of heat exchange unit is chosen, the function of the effectiveness is uniquely determined. For example, a countercurrent heat exchange unit is formulated as follows:

$$
\left.\begin{array}{l}
e=(k-1) /(k r-1) \\
k=\exp \left[a u(r-1) / w_{c}\right] \quad \text { and } \quad r \neq 1
\end{array}\right\}
$$

where $a$ and $u$ are the heat transfer area and the overall heat transfer coefficient, respectively. That $e$ includes the product of $a$ and $u$, which is denoted by $p$, is essential for the following discussion. This property is generally satisfied for all types of units.

Let us consider a network composed of $n$ streams with $m$ heat exchange units and some auxiliary heaters and/or coolers. No matter how complex a given network is, the system equations for each unit are all represented by Eq. (1). The auxiliary heat flow-rates are used as the manipulated variables to adjust the outlet temperature of some streams. Therefore, any outlet temperature from the network is linear not only in the inlet temperatures into the network, but also in the manipulated variables.

As the design specification each outlet temperature from the network is given by an inequality in this paper. Since each unit has three inequalities to take account of the minimum approach temperature and the direction of heat transfer, a design problem is represented by $n+3 m$ inequalities except the upper and lower bound conditions of the manipulated variable. For example, the total system constraints for the network in Fig. 1 are summarized in Appendix 1. The system constraints are generally represented as follows:

$$
g_{i}(\boldsymbol{T}, \boldsymbol{W}, \boldsymbol{E}, \boldsymbol{X}) \leqq 0, \quad i \in I
$$

where $\boldsymbol{T}, \boldsymbol{W}, \boldsymbol{E}$ and $\boldsymbol{X}$ are the vectors of inlet temperatures into the network, heat capacity flowrates of all streams, heat exchanger effectiveness of all units, and manipulated variables, respectively. $I$ is the index set of all the system constraints. The flexibility of a heat exchanger network is studied in relation to this type of system constraints.

\section{Property of the Feasible Regions}

\subsection{Feasible region in the $E$-space}

For a set of fixed values $\left(T^{*}, W^{*}\right)$ of inlet temperatures and heat capacity flow-rates of all streams, the feasible region $Q$ in the $\boldsymbol{E}$-space is defined as follows:

$$
Q=\left[\left.E\right|^{\exists} \boldsymbol{X}\left\{g_{i}\left(T^{*}, W^{*}, E, X\right) \leqq 0, i \in I\right\}\right]
$$

The expression above means that at any point $\boldsymbol{E}$ in the region $Q$ there must exist at least one value of $\boldsymbol{X}$ that satisfies all the system constraints for feasibility of operation.

As shown in Appendix 1 the constraint functions $g_{i}$ 's for the acyclic network are linear in $X$ and $e_{j}$ when the remaining $e_{k}$ 's are fixed.

$$
g_{i}=c_{i j}^{0}+c_{i j}^{1} e_{j}+(\boldsymbol{C}, \boldsymbol{X}) \leqq 0, \quad i \in I
$$

where $(\boldsymbol{C}, \boldsymbol{X})$ is the inner product of the manipulated variable vector and the constant vector and $c_{i j}^{*}(j=$ $0,1, \cdots, m)$ are the functions of $e_{k}$ 's $(k \neq j)$. When $e_{k}$ 's except $e_{j}$ are fixed the feasible region for all system constraints is polyhedral convex in the $\boldsymbol{X}-e_{j}$ space. Therefore, the region is connected.

For a network with loops such as the network in Fig. 2 the constraint functions $g_{i}$ 's are not linear in $e_{j}$, as shown in Appendix 2. In this case the connectedness of the feasible region in the $X-e_{j}$ space must be examined directly. The outline of investigation is also summarized in Appendix 2.

The projection of the connected feasible region in the $X-e_{j}$ space into the $e_{j}$ axis generates an interval. ${ }^{7}$ This implies that if in the $E$-space two different points on a one-dimensional manifold parallel to the $e_{j}$ axis are contained in the feasible region $Q$, then any point in the interval between these two points is also contained in $Q$. If this property is satisfied for each coordinate axis, then the feasible region $Q$ is called convex in coordinate directions or one-dimensional convex. But one should note that one-dimensional convexity does not mean connectedness.

\subsection{Feasible region in the $\boldsymbol{P}$-space}

The feasible region $Q^{\prime}$ in the $\boldsymbol{P}$-space is defined as follows:

$$
Q^{\prime}=\left[\left.\boldsymbol{P}\right|^{\exists} \boldsymbol{X}\left\{g_{i}\left(\boldsymbol{T}^{*}, \boldsymbol{W}^{*}, \boldsymbol{E}\left(\boldsymbol{P}, \boldsymbol{W}^{*}\right), \boldsymbol{X}\right) \leqq 0, i \in I\right\}\right]
$$

Since $p_{j}=a_{j} u_{j}$ is a continuous function of $e_{j}$, the feasible region in the $X-p_{j}$ space is also connected as well as in the $X-e_{j}$ space. ${ }^{7)}$ This means that the 


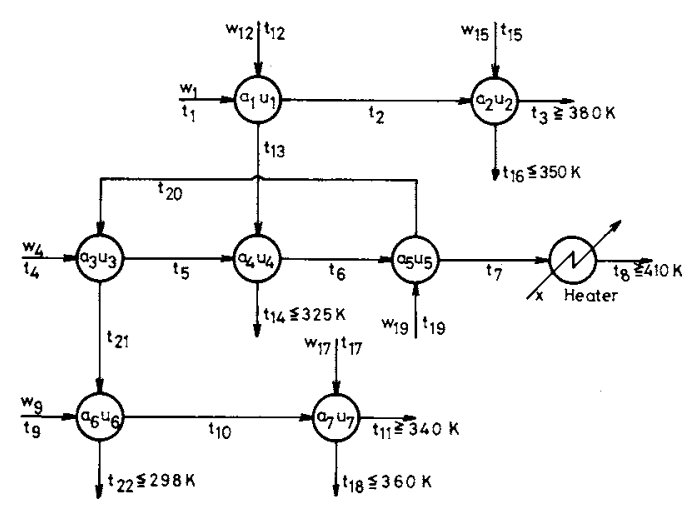

Fig. 2. A cyclic network with 7 countercurrent heat exchangers and a manipulated variable $x$ : example 2

feasible region $Q^{\prime}$ in the $\boldsymbol{P}$-space is also onedimensional convex. This region $Q^{\prime}$ is dependent on the values of $\boldsymbol{T}$ and $\boldsymbol{W}$, but independent of sizing of each heat exchange unit in the network.

\subsection{Feasible region in the $U$-space}

Sizing of all the heat exchange units in the network determines an inherent region $S$ of tolerance of the system for variations of vector $\boldsymbol{U}$ of all the overall heat transfer coefficients $u_{j}$ 's. Since $u_{j}$ is a continuous function of $p_{j}$, the feasible region in the $\boldsymbol{X}-u_{j}$ space is also connected. This means that the feasible region $S$ in the $U$-space is also one-dimensional convex.

\subsection{Region of required tolerance}

To cope with parameter variations in the $U$-space the region of required tolerance must be given in the same parameter space. This region can be determined on the basis of the subjective probability density function of the uncertain parameters. Usually, the region is given by an orthotope because the range of variation is defined separately for each parameter.

$$
D O=\left[\boldsymbol{U} \mid u_{j \min } \leqq u_{j} \leqq u_{j \max }, j \in M\right]
$$

where $M$ is the index set of all heat exchangers. A design specification for a flexible system is stated such that a network must be operable, i.e., there must exist $\boldsymbol{X}$, for any variation in the specified region $D O$.

\section{Problems Related to Flexibility}

When the inherent region $S$ of tolerance is onedimensional convex the feasibility test for the specified region $D O$ of required tolerance can be replaced by the feasibility test at every vertex of the orthotope $D O{ }^{1.2,5)}$ When a design $A^{*}$ is given, the magnitude of feasibility at a point $U$ in $D O$ is defined as follows: $:^{3,5,8)}$

$$
\begin{aligned}
& F\left(\boldsymbol{A}^{*}, \boldsymbol{U}\right) \\
& \quad=\operatorname{Min}_{v, \boldsymbol{X}}\left[v \mid g_{i}\left(\boldsymbol{T}^{*}, \boldsymbol{W}^{*}, \boldsymbol{E}\left(\boldsymbol{A}^{*}, \boldsymbol{U}\right), \boldsymbol{X}\right) \leqq v, i \in I\right]
\end{aligned}
$$

A positive value of $v$ means the quantity of relaxation

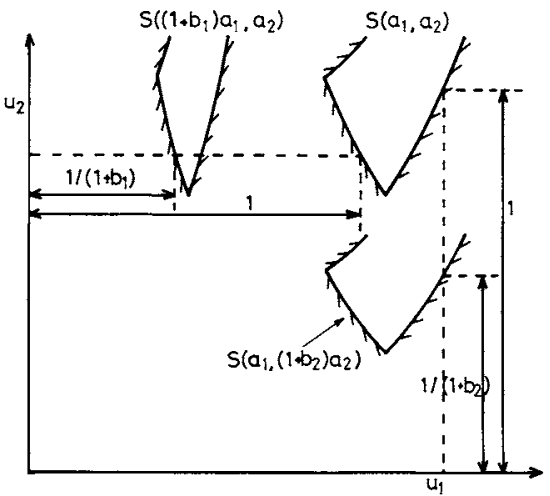

Fig. 3. Interaction between sizing of $a_{k}$ and location of the inherent region of tolerance in the uncertain parameter space

of all the constraints so that there exists at least one value of manipulated variable vector. Since the objective function $v$ and constraint functions $g_{i}$ 's are all linear in $v$ and $X$, the problem becomes a linear programming (LP) problem.

If there exists a vertex of $D O$ which gives a positive value to the function $F$, the inherent region $S$ of tolerance of the system does not contain the vertex. In other words, the point is an infeasible vertex. In this case the design $A^{*}$ must be changed so that the inherent region $S$ of tolerance of the redesigned system could include the infeasible vertex.

The following relationship holds between design revision and shift of the inherent region $S$ of tolerance:

"The change in $k$ th heat transfer area from $a_{k}$ to $a_{k}^{\prime}=$ $\left(1+b_{k}\right) a_{k}$ shifts a point $\boldsymbol{U}$ in $S$ to a new point $u_{k}^{\prime}=u_{k} /$ $\left(1+b_{k}\right)$, where $u_{j}^{\prime}=u_{j}(j \in M$ and $j \neq k)$." This fact is obvious because the temperature at any place in the network is invariant for the same value of $p_{k}=a_{k} u_{k}$. For example, when the heat transfer area $a_{1}$ is overdesigned by $b_{1} a_{1}$, any point in $S$ is shifted in the negative direction parallel to the $u_{1}$-axis as shown in Fig. 3. Later this property is successively used in the iterative design procedure to obtain a network with the specified region of tolerance.

On the other hand, the region of possible designs for the fixed values $U^{*}$ of parameters can be defined in the design-variable $\boldsymbol{A}$-space. The intersection of the regions for all vertices of the orthotope $D O$ is the region of possible design to guarantee the feasibility of operation, i.e. the existence of $\boldsymbol{X}$, for any variation in $D O$. If the intersection is empty, there exists no allocation of heat transfer areas under the specified configuration. Then the network must be changed. At the present time, heuristic rules are used for this purpose.

When a performance index from the economic viewpoint is represented by a function of design variables alone, a supporting hypersurface to the intersection in the $\boldsymbol{A}$-space gives an optimal design 
which satisfies the specification of system flexibility. The intersection of possible design is reduced as the magnitude of the orthotope becomes large. The tradeoff between the magnitude of required tolerance and the economic criterion can be obtained by repeating the optimal design for a different magnitude of the orthotope.

In the following section a noninferior design in the sense of minimizing every heat transfer area with the specified region of tolerance is discussed because of the uncertainty of the performance index.

\section{Iterative Design Method}

A noninferior design with the specified region of tolerance is obtained by the following iterative design method.

Step 1: Initial design Without taking account of parameter uncertainty the nominal design is calculated under the nominal set of values of the overall heat transfer coefficients.

Step 2: Feasibility test and redesign For the proposed design the magnitude of feasibility is calculated by Eq. (8) at every vertex of the specified orthotope. The magnitude of feasibility on each side of the orthotope is defined as follows:

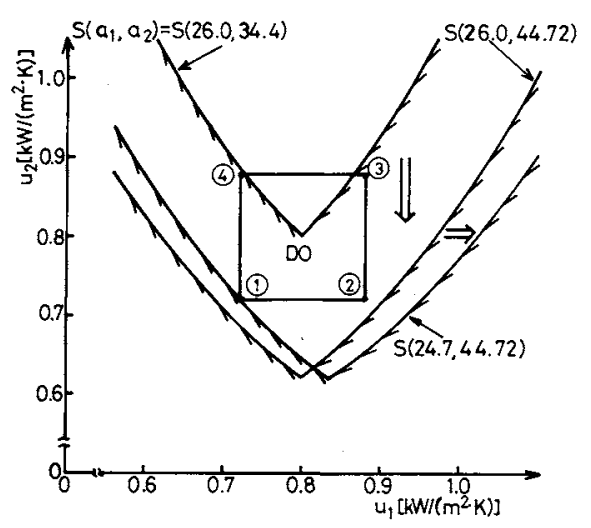

Fig. 4. Revising process in the uncertain parameter space: arrows show shift of the inherent region of tolerance

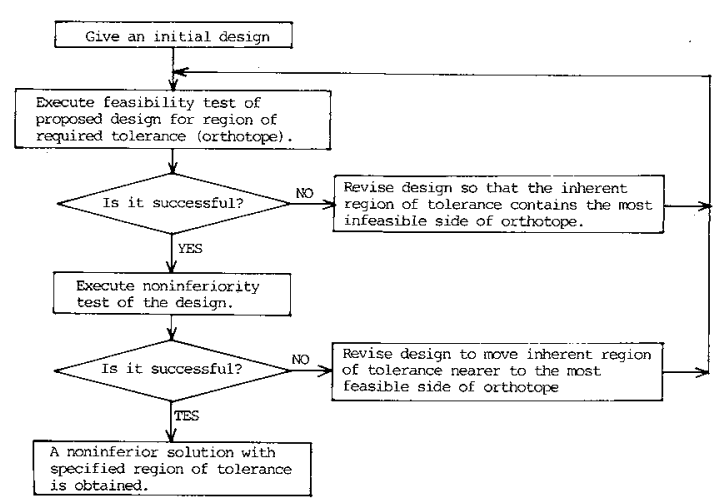

Fig. 5. Design procedure to obtain a noninferior heat exchanger network with the specified region of tolerance

$$
F S_{i}\left(A^{*}, \boldsymbol{U}\right)=\sum_{\boldsymbol{U} \in V \boldsymbol{S}_{i}} F\left(A^{*}, \boldsymbol{U}\right)
$$

where $V S_{i}$ is the set of all $2^{m-1}$ vertices on $i$ th side of the orthotope. Each side is denoted by the plane which includes itself, i.e. $u_{j}=u_{j \min }$ or $u_{j \max }$. Among all $2 m$ sides of $D O$ the two sides which give the maximum and minimum values to $F S_{i}$ are useful to determine the element of $\boldsymbol{A}$ for redesign. The former corresponds to the least feasible (the most infeasible) one and the latter corresponds to the most feasible (the least infeasible) one.

When at least one infeasible vertex exists, a shift of the inherent region $S$ of tolerance to contain the most infeasible side (the least feasible one) is attempted by overdesign or underdesign of the element perpendicular to the side (Rule 1). The relationship between design revision and shift of the region $S$ can be applied for this purpose.

When no infeasible vertex exists, the corresponding design has the specified region of tolerance. However, the design may have much more tolerance than a designer wants. Therefore, noninferiority of the design is tested in the next step.

Step 3: Noninferiority test Useless overdesign should be avoided by successive improvement. A smaller heat transfer area is preferable for each unit on condition that the design has the required tolerance. Therefore, a noninferior solution is searched in the sense of minimizing every heat transfer area.

If the sides that contain the lowest point $\left\{u_{j}=\right.$ $\left.u_{j \min }, j \in M\right\}$ have the margin to the in herent regions $S$ of tolerance as shown in Fig. 4, then some heat transfer areas can be decreased. Among these sides the most feasible one that has the biggest margin to $S$ is found. It is denoted by $\left\{u_{k}=u_{\mathrm{kmin}}\right\}$. Then a shift of the inherent regions $S$ of tolerance nearer to the side is achieved by decreasing $k$ th element of $\boldsymbol{A}$ (Rule 2).

When a vertex of $D O$ lies on the boundary of $S$, a direction of shift for further improvement is searched by decreasing each area slightly. This improvement is repeated until no possible shift exists. The final design lies on the noninferior solution set of possible design in the $\boldsymbol{A}$-space. The procedure to obtain a noninferior design with the specified region of tolerance is summarized in Fig. 5.

\section{Illustrative Examples}

Example 1 Acyclic network The optimal design problem of the heat exchanger network in Fig. 1 was studied with uncertainty in the overall heat transfer coefficient of the countercurrent heat exchange unit. The outlet temperatures of two cold streams and one hot stream must satisfy inequalities shown in the network. All the system constraints are represented by 11 inequalities with a manipulated variable of the heat 
flow-rate in the auxiliary heater. The design data for the network are summarized in Table 1. The region of required tolerance is given by the following orthotope.

$$
D O=\left[U \mid 0.9 u_{j}^{N} \leqq u_{j} \leqq 1.1 u_{j}^{N}, j \in\{1,2\}\right]
$$

The initial design was obtained by minimizing the total heat transfer area for the nominal set of values of the overall heat transfer coefficients. This design does not have enough tolerance, as shown in Fig. 4. All four vertices $1-4$ are infeasible. Then rule 1 was applied to widen the inherent region of tolerance. After three increases of 10 percent in $a_{2}$ the orthotope $D O$ was contained in the inherent region $S$ of toler-

Table 1. Design data and results for example 1

\section{Design data}

(1) Nominal values of parameters:

$$
\begin{array}{lll}
t_{1}=420 \mathrm{~K} & w_{1}=30 \mathrm{~kW} / \mathrm{K} & u_{1}=0.8 \mathrm{~kW} /\left(\mathrm{m}^{2} \cdot \mathrm{K}\right) \\
t_{4}=385 \mathrm{~K} & w_{4}=10 \mathrm{~kW} / \mathrm{K} & u_{2}=0.8 \mathrm{~kW} /\left(\mathrm{m}^{2} \cdot \mathrm{K}\right) \\
t_{6}=480 \mathrm{~K} & w_{6}=15 \mathrm{~kW} / \mathrm{K} &
\end{array}
$$

(2) Bounds of manipulated variable: $0 \leqq x \leqq 3000 \mathrm{~kW}$

(3) Minimum approach temperature of each heat exchanger: $\Delta t=0 \mathrm{~K}$

\begin{tabular}{lccc}
\hline Results & & & \\
\hline & & Number of & Heat transfer areas $\left[\mathrm{m}^{2}\right]$ \\
\cline { 4 - 4 } Alternative designs & & $a_{1}$ & $a_{2}$ \\
\hline revision & 0 & 26.0 & 34.4 \\
Initial design & 3 & 26.0 & 44.72 \\
$\begin{array}{l}\text { Design with fixed } \\
\text { tolerance }\end{array}$ & & & 44.72 \\
Noninferior design & 7 & 24.7 & \\
$\quad$ with fixed tolerance & & & \\
\end{tabular}

ance. But this design has excessive flexibility. Several revisions by rule 2 with variable scaling gave a noninferior design, which is shown in Table 1 . The revising process in the uncertain-parameter space and in the design-variable space are illustrated in Figs. 4 and $\mathbf{6}$, respectively. The CPU-time needed to obtain the noninferior design was 5 seconds by the NEC ACOS-1000 computer system.

Example 2 Cyclic network Sizing of the heat exchanger network with seven countercurrent heat exchange units shown in Fig. 2 was studied on condition that the network has the required tolerance. The outlet temperatures of three cold streams and four hot streams must satisfy the inequalities shown in the network. All the system constraints are represented by 30 inequalities including a manipulated variable of the heat flow-rate in the heater. The design data for the

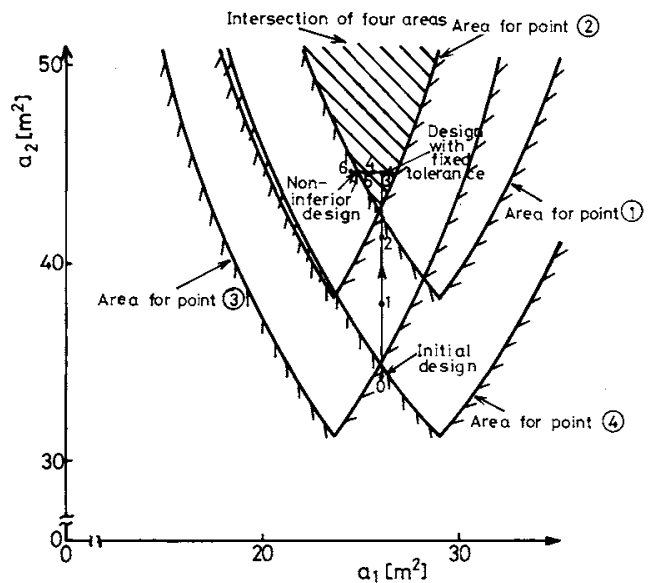

Fig. 6. Revising process in the design variable space: points 1-4 correspond to the vertices in the uncertain parameter space in Fig. 4

Table 2. Design data and results for example 2

Design data

(1) Nominal values of parameters:

$\begin{array}{lll}t_{1}=310 \mathrm{~K} & w_{1}=5 \mathrm{~kW} / \mathrm{K} & u_{1}=0.8 \mathrm{~kW} /\left(\mathrm{m}^{2} \cdot \mathrm{K}\right) \\ t_{4}=290 \mathrm{~K} & w_{4}=6 \mathrm{~kW} / \mathrm{K} & u_{2}=0.8 \mathrm{~kW} /\left(\mathrm{m}^{2} \cdot \mathrm{K}\right) \\ t_{9}=285 \mathrm{~K} & w_{9}=2 \mathrm{~kW} / \mathrm{K} & u_{3}=0.8 \mathrm{~kW} /\left(\mathrm{m}^{2} \cdot \mathrm{K}\right) \\ t_{12}=400 \mathrm{~K} & w_{12}=4 \mathrm{~kW} / \mathrm{K} & u_{4}=0.8 \mathrm{~kW} /\left(\mathrm{m}^{2} \cdot \mathrm{K}\right) \\ t_{15}=450 \mathrm{~K} & w_{15}=2 \mathrm{~kW} / \mathrm{K} & u_{5}=0.8 \mathrm{~kW} /\left(\mathrm{m}^{2} \cdot \mathrm{K}\right) \\ t_{17}=400 \mathrm{~K} & w_{17}=2 \mathrm{~kW} / \mathrm{K} & u_{6}=0.8 \mathrm{~kW} /\left(\mathrm{m}^{2} \cdot \mathrm{K}\right) \\ t_{19}=430 \mathrm{~K} & w_{19}=2.5 \mathrm{~kW} / \mathrm{K} & u_{7}=0.8 \mathrm{~kW} /\left(\mathrm{m}^{2} \cdot \mathrm{K}\right)\end{array}$

\begin{tabular}{|c|c|c|c|c|c|c|c|c|}
\hline \multirow{2}{*}{ Alternative designs } & \multirow{2}{*}{$\begin{array}{l}\text { Number of } \\
\text { revision }\end{array}$} & \multicolumn{7}{|c|}{ Heat transfer areas $\left[\mathrm{m}^{2}\right]$} \\
\hline & & $a_{1}$ & $a_{2}$ & $a_{3}$ & $a_{4}$ & $a_{5}$ & $a_{6}$ & $a_{7}$ \\
\hline Initial design & 0 & 3.34 & 8.11 & 4.14 & 7.80 & 6.78 & 3.28 & 1.67 \\
\hline Design with fixed tolerance & 16 & 3.67 & 12.16 & 4.14 & 8.58 & 9.49 & 3.94 & 2.17 \\
\hline $\begin{array}{l}\text { Noninferior design with } \\
\text { fixed tolerance }\end{array}$ & 27 & 3.67 & 12.00 & 4.01 & 8.38 & 9.49 & 3.94 & 2.03 \\
\hline
\end{tabular}

(2) Bounds of manipulated variable: $0 \leqq x \leqq 1000 \mathrm{~kW}$

(3) Minimum approach temperature of each heat exchanger: $\Delta t=0 \mathrm{~K}$

Results 
network are summarized in Table 2. The region of required tolerance is given by the following orthotope.

$$
D O=\left[U \mid 0.9 u_{j}^{N} \leqq u_{j} \leqq 1.1 u_{j}^{N}, j \in\{1,2, \cdots, 7\}\right]
$$

The design revision process is summarized in Table 2. A noninferior design with the feasibility for any variation in the orthotope $D O$ was obtained after 27 revisions. The total CPU-time to solve this problem was 166 seconds by the ACOS-1000. This result implies that the proposed design procedure will be useful in solving practical problems.

\section{Conclusion}

In a heat exchanger network the uncertainty in overall heat transfer coefficients is one of the most important issues at the stage of design. By geometric consideration of the feasible region in the uncertainparameter space a simple relationship between sizing and the inherent region of tolerance was found. This property is valid for both acyclic and cyclic networks and can be applied to the design of a heat exchanger network with a specified region of required tolerance. A noninferior network in the sense of minimizing every heat transfer area was effectively obtained by the proposed design method.

Appendix 1. Total system constraints for the network in Fig. 1:

$$
\begin{aligned}
& g_{1}=500-t_{2}-x / w_{1} \leqq 0 \\
& g_{2}=430-t_{5} \leqq 0 \\
& g_{3}=t_{8}-410 \leqq 0 \\
& g_{4}=t_{1}-t_{6} \leqq 0 \\
& g_{5}=\Delta t-t_{6}+t_{2} \leqq 0 \\
& g_{6}=\Delta t-t_{7}+t_{1} \leqq 0 \\
& g_{7}=t_{4}-t_{7} \leqq 0 \\
& g_{8}=\Delta t-t_{7}+t_{5} \leqq 0 \\
& g_{9}=\Delta t-t_{8}+t_{4} \leqq 0 \\
& g_{10}=-x \leqq 0 \\
& g_{11}=x-\bar{x} \leqq 0
\end{aligned}
$$

where $\Delta t$ is the minimum approach temperature.

The temperatures $t_{2}, t_{5}, t_{7}$ and $t_{8}$ are represented by the linear forms of the inlet temperatures into the network $t_{1}, t_{4}$ and $t_{6}$ as follows:

$$
\begin{aligned}
& t_{2}=\left(1-e_{1}\right) t_{1}+e_{1} t_{6} \\
& t_{7}=e_{1} r_{1} t_{1}+\left(1-e_{1} r_{1}\right) t_{6} \\
& t_{5}=\left(1-e_{2}\right) t_{4}+e_{2} e_{1} r_{1} t_{1}+e_{2}\left(1-e_{1} r_{1}\right) t_{6} \\
& t_{8}=e_{2} r_{2} t_{4}+\left(1-e_{2} r_{2}\right) e_{1} r_{1} t_{1}+\left(1-e_{2} r_{2}\right)\left(1-e_{1} r_{1}\right) t_{6}
\end{aligned}
$$

where

$$
0 \leqq e_{j} \leqq 1 \quad(j=1,2)
$$

Appendix 2. The connectedness of the feasible region in the $X-e_{j}$ space for the network in Fig. 2:

This network has a loop of $t_{20}-t_{5}-t_{6}-t_{20}$, on which the temperatures must be solved simultaneously. The constraint with the manipulated variable is only one for the outlet temperature of $t_{8}$, i.e.,

$$
g_{1}=410-t_{7}-x / w_{4} \leqq 0
$$

where

$$
\begin{aligned}
t_{7}= & \left\{\left(1-e_{3}\right)\left(1-e_{4}\right)\left(1-e_{5}\right) t_{4}+e_{4}\left(1-e_{5}\right) t_{13}\right. \\
& \left.+\left[e_{5}+e_{3}\left(1-e_{4}\right)\left(1-e_{5}-e_{5} r_{5}\right) t_{19}\right]\right\} /\left[1-e_{3}\left(1-e_{4}\right) e_{5} r_{5}\right]
\end{aligned}
$$

When all $e_{k}$ 's except $e_{j}$ are fixed the above inequality is rewritten as follows:

$$
g_{1}=c_{1 j}^{0}+c_{1 j}^{1} /\left(c_{1 j}^{2} e_{j}-1\right)-x / w_{4} \leqq 0
$$

where $c_{1 j}^{i}$ 's $(i=0,1,2)$ are the functions of $e_{k}$ 's $(k \neq j)$. This inequality makes a connected region in $e_{j}-\boldsymbol{X}$ space with $0 \leqq e_{j} \leqq 1$. It is either convex or nonconvex. Since the remaining constraints do not include the manipulated variable, they are all represented as follows:

$$
\underline{e}_{j} \leqq e_{j} \leqq \bar{e}_{j}
$$

Consequently, the set of all inequalities generates a connected region in $e_{j}-X$ space. This property is satisfied for any $j$.

\section{Nomenclature}

$\begin{array}{llr}a_{j} & =\text { heat transfer area } & {\left[\mathrm{m}^{2}\right]} \\ A & =\text { vector of } a_{j} & {[-]} \\ b & =\text { scaling factor in design revision } & {[-]} \\ c_{i j}^{*} & =\text { coefficient in Eq. (5) } & {[-]} \\ C & =\text { constant vector in Eq. (5) } & {[-]} \\ D O & =\text { orthotope } & {[-]} \\ e_{j} & =\text { heat exchanger effectiveness } & {[-]} \\ \boldsymbol{E} & =\text { vector of } e_{j} & {[-]} \\ F & =\text { magnitude of feasibility at a point } & {[-]} \\ F S & =\text { magnitude of feasibility on a side } & {[-]} \\ g_{i} & =\text { system constraint function } & {[-]} \\ p_{j} & =\text { product of } a_{j} \text { and } u_{j} & {[\mathrm{~kW} / \mathrm{K}]} \\ \boldsymbol{P} & =\text { vector of } p_{j} & {[-]} \\ Q & =\text { feasible region in } \boldsymbol{E} \text {-space } & {[-]} \\ Q^{\prime} & =\text { feasible region in } \boldsymbol{P} \text {-space } & {[-]} \\ r_{j} & =\text { ratio of heat capacity flow rates } & {[-]} \\ S & =\text { feasible region in } U \text {-space } & {[-]} \\ T & =\text { vector of inlet temperatures } & {[\mathrm{K}]} \\ u_{j} & =\text { overall heat transfer coefficient } & {\left[\mathrm{kW} / \mathrm{m}^{2} \mathrm{~K}\right]} \\ U & =\text { vector of } u_{j} & {[-]} \\ V S & =\text { set of all vertices on a side } & {[-]} \\ \boldsymbol{W} & =\text { vector of heat capacity flow rates } & {[\mathrm{kW} / \mathrm{K}]} \\ X & =\text { vector of manipulated variables } & {[-]} \\ & & \end{array}$

\section{〈Superscript〉}

$$
\begin{array}{ll}
c & =\text { cold stream } \\
h & =\text { hot stream } \\
i & =\text { inlet } \\
N & =\text { nominal value } \\
o & =\text { outlet }
\end{array}
$$

\section{Literature Cited}

1) Bandler, J. W.: J. Optimiz. Theory \& Appl., 14, 99 (1974).

2) Bandler, J. W., P. C. Liu and J. H. K. Chen: IEEE Trans. Microwave Theory \& Tech., MTT-23, 630 (1975).

3) Grossmann, I. E. and C. Floudas: I. Chem. E. Symp., No. 92 , 619 (1985).

4) Grossmann, I. E. and M. Morari: Proc. of 2nd International Conference on Foundations of Computer-Aided Process Design, p. 931, CACHE (1984).

5) Halemane, K. P. and I. E. Grossmann: AIChE J., 29, 425 (1983). 
6) Kunugita, E., H. Nishitani and Y. Kutsuwa: I. Chem. E. Symp., No. 92, 631 (1985).

7) Simmons, G. F.: Introduction to Topology and Modern Analysis, Chapt. 6, McGraw-Hill (1963).
8) Swaney, R. E. and I. E. Grossmann: AIChE J., 31, 621 (1985).

9) Takamatsu, T., I. Hashimoto and H. Nishitani: Kagaku Kōgaku, 38, 309 (1974)

\title{
EFFECT OF A RADIATION SHIELD ON MELT/CRYSTAL INTERFACE SHAPE AND PULL RATE OF SILICON CZ PULLER
}

\author{
TAKAO TSUKADA, NOBUYUKI IMAISHI AND MITSUNORI HOZAWA \\ Chemical Research Institute of Non-Aqueous Solutions, Tohoku University, Sendai 980
}

\begin{abstract}
Key Words: Crystal Growth, Silicon, Czochralski Method, Radiation Shield, Heat Conduction, Radiation, Interface Shape, Numerical Simulation

For a silicon CZ crystal puller, the effect of an annular radiation shield on the temperature profile in the melt and crystal and on the shape of the melt/crystal interface was studied theoretically by use of the finite element analysis based on the conduction-dominated model.

It was found that a radiation shield of an appropriate shape and located at an optimum position in the furnace can make the interface shape less convex to the crystal and can reduce the input power to the furnace at all stages of crystal growth in comparison with operation without a shield. Also, use of a radiation shield increases the pull rate, i.e. the productivity of the $\mathrm{CZ}$ puller, because the axial temperature gradient near the melt/crystal interface becomes steeper.
\end{abstract}

\section{Introduction}

In the Czochralski (CZ) method of growing single crystals for use in semiconductor devices, the shape of the melt/crystal interface should be kept as.flat as possible during crystal growth in order to produce radially homogeneous wafers. As the macroscopic melt/crystal interface corresponds to the meltingpoint isotherm, the interface shape is influenced by thermal conditions such as crucible temperature, crystal pull rate or ambient temperature. Accordingly, control of the quality of crystals requires knowledge of the heat transfer mechanism in the melt and the crystal, and of the shape of the melt/crystal interface for various growing conditions. A number of theoretical studies of this problem have been carried out. ${ }^{1,2,6,8,13)}$ In these analyses, however, the melt/ crystal interface is assumed to be flat. Recently, finite element analyses ${ }^{3-5,7,10,12)}$ based on the conductiondominated model have been developed to determine simultaneously the temperature distributions and the melt/crystal interface shape. Though the heat transfer

Received November 5, 1987. Correspondence concerning this article should be addressed to T. Tsukada. in the melt and the interface shape are influenced by convection in the melt, those for a system with small Prandtl number, such as silicon, can be well approximated by this model. The interface shape is, however, not widely controllable by varying the thermal conditions, such as crucible temperature, pull rate or ambeint temperature. For example, the interface shape of a crystal of given radius is made flatter by increasing crucible temperature and decreasing pull rate at the same time. ${ }^{12)}$ From the industrial point of view, however, higher-velocity pulling is required. Thus, the development of a new idea which allows control of the interface shape without reducing productivity is required. Srivastava et al. ${ }^{11)}$ proposed that the interface shape can be widely controlled by blowing an inert gas jet at a high flow rate onto the crystal surface near the bottom of the crystal. This is a typical "active control" of interface shape. Such an active method may provide a wide range of control. However, interface shape is highly sensitive to gas flow rate, position of the nozzle and so on. And the melt/gas interface is highly disturbed. Thus such an active method does not seem applicable to the industrial $\mathrm{CZ}$ puller.

We reported in a previous work ${ }^{12)}$ that a radiation 